\section{JTI}

JOURNAL OF

TRAUMA AND INJURY

\title{
Calcaneal Fractures: A Soft Tissue Emergency
}

\author{
Tae-Seong Kim, M.D. ${ }^{1,2}$, Chang-Wug Oh, M.D., Ph.D. ${ }^{1,2}$, Joon-Woo Kim, M.D., Ph.D. ${ }^{1,2}$, \\ Kyung-Hyun Park, M.D. ${ }^{1,2}$ \\ ${ }^{1}$ Regional Trauma Center, Kyungpook National University Hospital, Daegu, Korea \\ ${ }^{2}$ Department of Orthopaedic Surgery, School of Medicine, Kyungpook National University, \\ Daegu, Korea
}

Received: May 31, 2018

Revised: July 19, 2018

Accepted: August 6, 2018

\section{Correspondence to}

Chang-Wug Oh, M.D., Ph.D.

Department of Orthopedic Surgery, Kyungpook National University Hospital, School of Medicine, Kyungpook National University, 130 Dongdeok-ro, Jung-gu,

Daegu 41944, Korea

Tel: $+82-53-420-5630$

Fax: $+82-53-422-6605$

E-mail:cwoh@knu.ac.kr
Calcaneal fractures are quite often seen in patients with axial loading injury. In the tongue-type of calcaneusal fractures or tuberosity avulsion fractures, bone fragments are often superiorly and posteriorly displaced, because of the insertion of the Achilles tendon and pull of the gastroc-soleus complex. The Ddisplaced bone fragment compresses the soft tissues, leading tothat makes skin necrosis. To prevent further soft tissue injury, early recognition of the injury by the emergency physician and immediate orthopedic consultation is needed.

Keywords: Calcaneus; Heel; Necrosis

\section{INTRODUCTION}

Calcaneal fractures represent the most common tarsal bone fracture [1]. Irrespective of whether patients with calcaneal fractures are treated with splint immobilization and sent for outpatient follow-up or transferred to the orthopedic service for open reduction internal fixation, morphological variants of calcaneal fractures, including tonguetype fractures and associated tuberosity avulsion fractures, represent a unique pattern of injury to the bone and soft tissue [2]. Occasionally, the diagnosis is either missed or the treatment is delayed in certain situations, which causes skin necrosis on the heel. To optimize patient outcomes and to reduce misdiagnosis, appropriate evaluation and treatment should begin in the emergency department.

This is an Open Access article distributed under the terms of the Creative Commons Attribution Non-Commercial License (http://creativecommons.org/licenses/by-nc/4.0/) which permits unrestricted noncommercial use, distribution, and reproduction in any medium, provided the original work is properly cited. 


\section{CASE REPORT}

\section{Case 1}

A 76-year-old man sustained an injury while riding a motorcycle. He had left femur intertrochanteric fracture, right distal femur condyle fracture, right distal tibiofibular fracture, and right calcaneal fracture. Following the initial resuscitation, bridging external fixation was performed for the long bone fractures as damage control surgery. The patient had to be treated in an intensive care unit for a while because his condition was unstable. $\mathrm{He}$ complained of pain in his right heel during this period. Radiographs of his calcaneus showed calcaneal tuber-

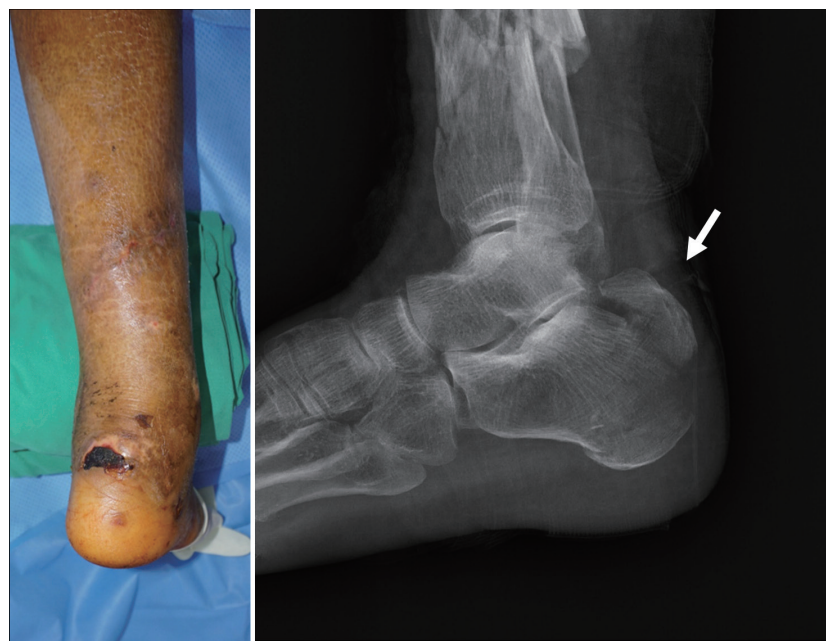

Fig. 1. Displaced calcaneal tuberosity fragment can compress the heel (arrow), which can cause skin necrosis. osity avulsion fracture with superior displacement that was compressing the skin posteriorly. Surgical correction could not be planned immediately owing to the patient's unstable general condition; only a plantar flexion splint could be fixed. The patient recovered after 3 weeks, but his heel had developed necrotic changes (Fig. 1). Before open reduction and internal fixation could be performed, soft tissue intervention was required. A reverse sural artery flap was constructed for skin coverage. After the flap wound had healed, open reduction and internal fixation was performed. At 1-year follow-up, the bone had united, and the patient could walk without a cane (Fig. 2).

\section{Case 2}

A 60-year-old man sustained an injury after falling from a

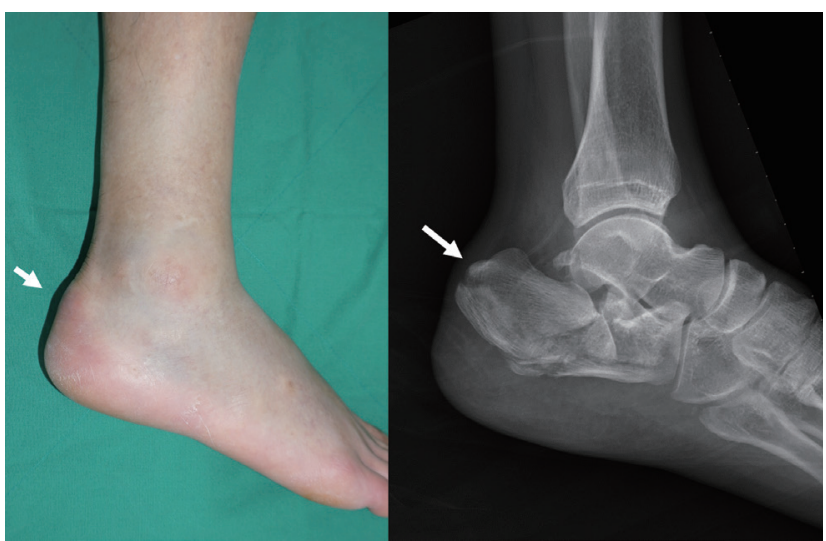

Fig. 3. Displaced fragment causes skin tenting on the posterior heel (arrows).
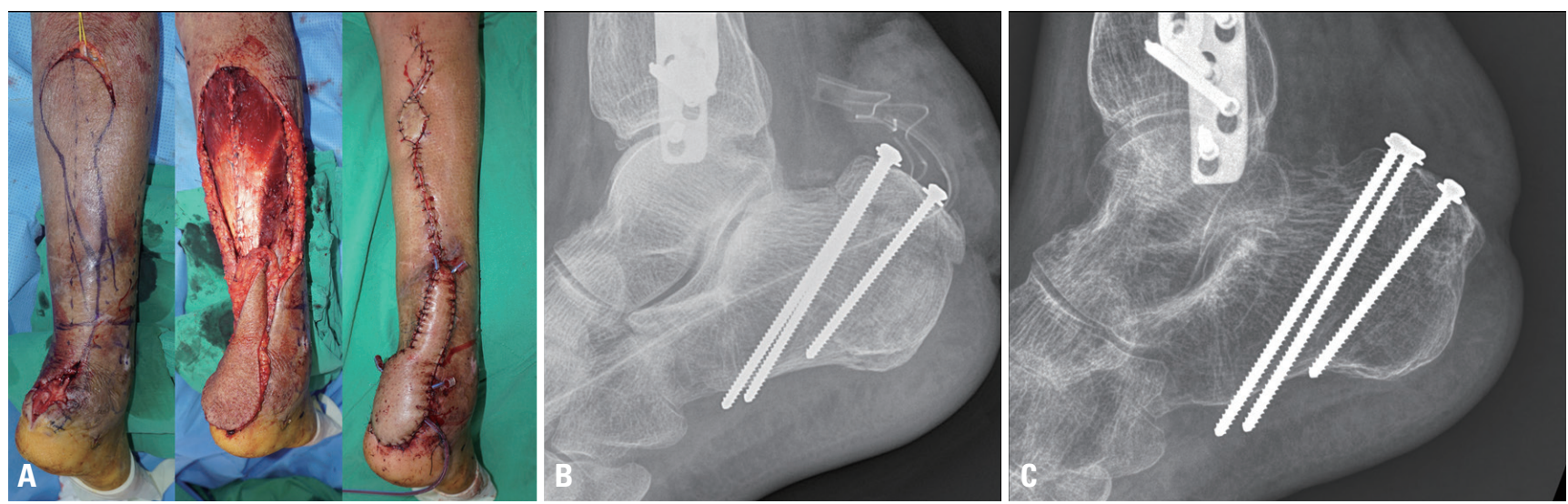

Fig. 2. (A) Reverse sural artery flap is a very useful local rotation flap that can cover the skin defect around the ankle joint. (B) Postoperative X-ray shows tuberosity avulsion fragment fixed by three $3.5 \mathrm{~mm}$ cortical screw with washers. (C) One year after, bone has united without any complications. 


\section{JTI}

height of 6 meters. He reported landing on his heels, with immediate pain in both his heels. He was seen at another hospital, where radiographic examination was performed and he was told that he had fractures of bones in both his heels. He was transferred to our hospital after he was immobilized in a short-leg posterior mold splint with vvthe vankle in a neutral position. On physical examination following the splint removal, he was found to have significant tenderness over his right heel. His neurovascular examination was normal. Radiographs of his foot were obtained, which showed a tongue-type calcaneal fracture in the right foot, with superior displacement that was tenting the skin posteriorly (Fig. 3): this meant a higher likelihood of skin necrosis. Local damage control surgery was performed the same day he presented in the emergency department. Simple leverage technique was employed in the surgery, and the fragment was fixed using several K-wires, without any soft tissue intervention. After 2 weeks, when the soft tissue swelling had subsided, open reduction and internal fixation was performed via the extensile lateral approach. At 7 months follow-up, the bone had united without any complications, and the patient had recovered good function of the limb (Fig. 4).

\section{DISCUSSION}

Calcaneal fractures are commonly diagnosed and account for $60 \%$ of all tarsal fractures [1]. These fractures frequently occur following a high-energy axial load to the heel, although even relatively low-energy trauma $[3,4]$ may cause them. Nonsurgical treatment may be recommended if the pieces of broken bone have not been displaced by the force of the injury. A cast, splint, or brace will hold the bones in proper position while they heal. The patient may have to wear a cast for 6 to 8 weeks or even longer. During this time, the patient is not permitted any weight bearing on the joint until the bone is completely healed. If the bones are displaced, surgical intervention may be recommended. Displaced fractures of the calcaneus are the result of high-energy axial loading injuries. Consequently, the damage to the surrounding soft tissue results in significant swelling. Fracture-blister formation is common. To minimize soft tissue complications during the preoperative period, the foot should be elevated to the level of the heart and immediately splinted with the ankle in neutral position. Surgical timing is dependent on the condition of the soft tissues. Swelling should be decreased such that tissue turgor allows skin wrinkling in response to gentle pressure. Operating through excessively swollen tissue has been shown to significantly increase the risk of wound healing problems and infection. For this reason, it is now a standard practice to wait until the soft tissue swelling has subsided, often 1-2 weeks or more following the injury. In contrast, in the case of a tonguetype calcaneal fracture (Fig. 5) or a calcaneal tuberosity avulsion fracture, the fracture fragment can compress the soft tissue of the posterior ankle, which can lead to soft

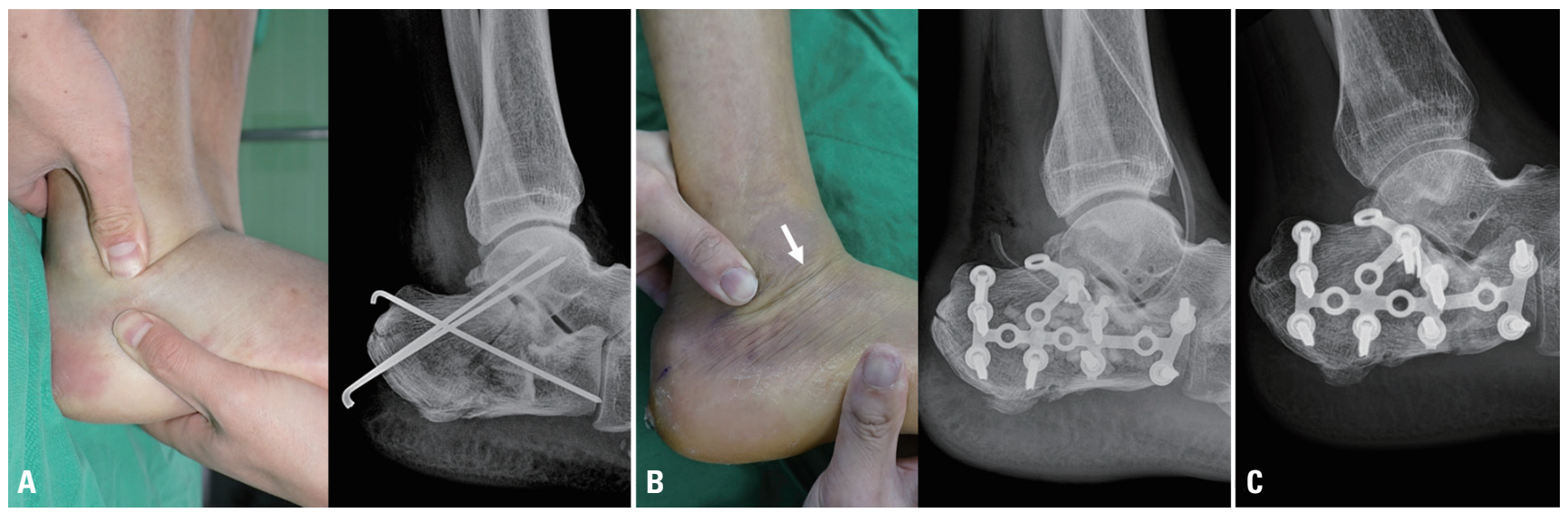

Fig. 4. (A) On the admission day, there is no wrinkle sign because of soft tissue swelling. (B) After soft tissue swelling subsided, with eversion and dorsiflexion of ankle joint, wrinkle sign can be seen (arrow). This means a lower risk of skin problems. (C) One-year follow-up, bone has united. 
tissue problems. There are three types of avulsion fractures of the calcaneal tuberosity: type I is a sleeve fracture in which a small piece of cortical bone is avulsed; type II is a beak fracture with an oblique fracture line that exits close but posterior to the posterior facet; and type III is an infrabursal fracture that is an avulsion off the middle third of the posterior aspect of the tuberosity. Type II fractures may require prompt reduction. To prevent these complications, it is recommended that the injured ankle be splinted in the plantar flexion position to minimize soft tissue tension, thereby reducing soft tissue devitalization. Otherwise the displaced bone fragment can tent the skin of the posterior heel, eventually leading to skin

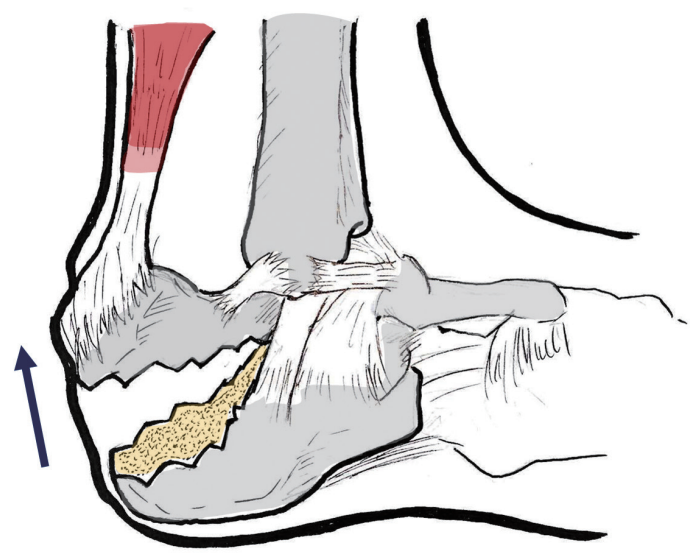

Fig. 5. Tongue-type calcaneal fractures are longitudinal fractures that involve the calcaneal tuberosity. This fragment is commonly displaced superiorly and posteriorly, because of rotation of the fragment from the pull of the gastrocnemius-soleus complex. necrosis [4-6]. Gardner et al. [3] reported the presence of posterior skin tenting in $21 \%$ of 139 tongue-type fractures. Soft tissue coverage or even amputation was needed in $29 \%$ of these patients. When there is a threat of tenting of the posterior skin, ankle plantar flexion splint is recommended. However, a plantar flexion splint alone may not always be sufficient to reduce the bone fragment. In cases where the bone fragment still tents the skin even after applying the plantar flexion splint, local damage control surgery is needed. Several authors have proposed using an external fixator system for damage control surgery for the calcaneus: inserting the external fixator ensures that the morphology of the calcaneus is restored and helps the soft tissues return to normal more quickly $[7,8]$. However, the external fixator is quite bulky, and it may be uncomfortable for the patient to move. Further, it is difficult to firmly fix the bone fragment by external fixation alone. Unlike the external fixator, our technique is simple, and the patients are also relatively comfortable with our technique. With the simple leverage technique using the Schanz screw pin, the bone fragment can be reduced easily, making only a small incision on the posterior aspect of the ankle. Temporary fixation is achieved with several pins until the soft tissue swelling subsides (Fig. 6), following which the patient undergoes definite open reduction and internal fixation. However, the most important point is early diagnosis. In cases where the displaced fragment tents the posterior ankle, early detection and immediate treatment are required.

In conclusion, tongue-type calcaneal fractures or cal-
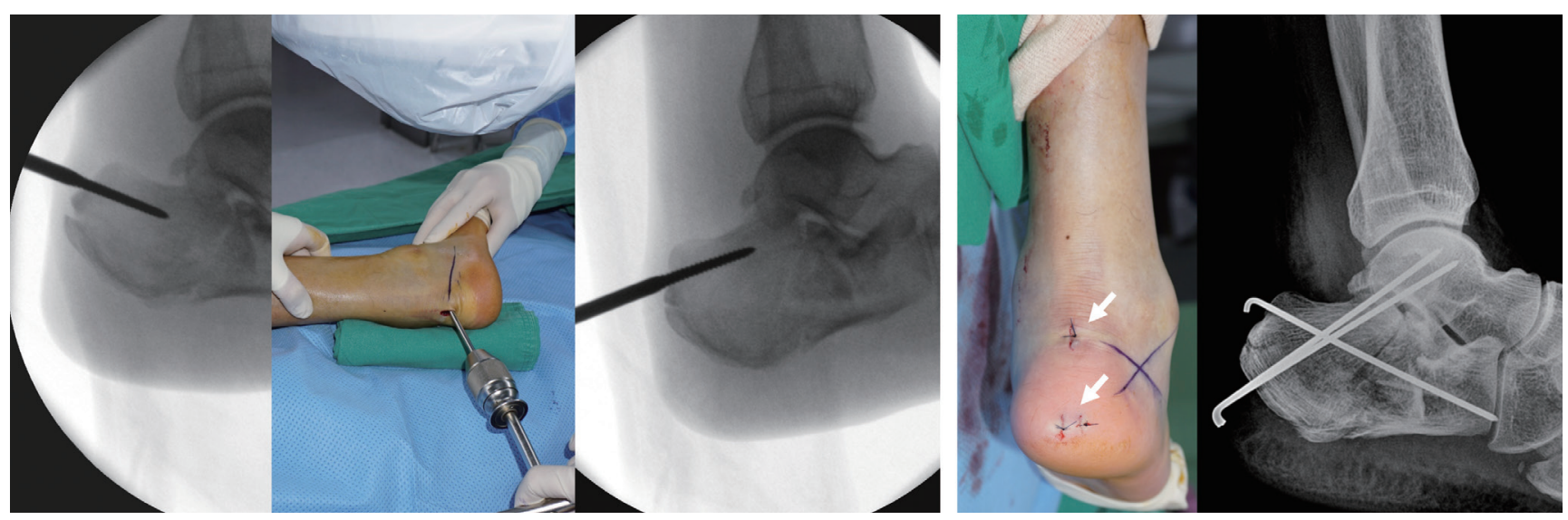

Fig. 6. Leverage technique with simple stab incision (arrows), reduces superiorly displaced bone fragment easily. 
caneal avulsion fracture with a displaced tuberosity can harm the soft tissues. Early recognition by the emergency physician can affect patient outcome. Whenever a calcaneal fracture with skin at risk is identified, the orthopedic service should be consulted as soon as possible.

\section{REFERENCES}

1. Simon RR, Sherman SC. Emergency orthopedics. 6th ed. New York:McGraw-Hill;2011.

2. Essex-Lopresti P. The mechanism, reduction technique, and results in fractures of the os calcis. Br J Surg 1952;39:395-419.

3. Gardner MJ, Nork SE, Barei DP, Kramer PA, Sangeorzan BJ, Benirschke SK. Secondary soft tissue compromise in tongue-type calcaneus fractures. J Orthop Trauma 2008;22:439-45.
4. Hess M, Booth B, Laughlin RT. Calcaneal avulsion fractures: complications from delayed treatment. Am J Emerg Med 2008;26:254.e1-4.

5. Squires B, Allen PE, Livingstone J, Atkins RM. Fractures of the tuberosity of the calcaneus. J Bone Joint Surg Br 2001;83:55-61.

6. Watson TS. Soft tissue complications following calcaneal fractures. Foot Ankle Clin 2007;12:107-23.

7. Baumgaertel FR, Gotzen L. Two-stage operative treatment of comminuted os calcis fractures. Primary indirect reduction with medial external fixation and delayed lateral plate fixation. Clin Orthop Relat Res 1993;(290):132-41.

8. Besch L, Waldschmidt JS, Daniels-Wredenhagen M, Varoga D, Mueller M, Hilgert RE, et al. The treatment of intra-articular calcaneus fractures with severe soft tissue damage with a hinged external fixator or internal stabilization: long-term results. J Foot Ankle Surg 2010;49:8-15. 